\title{
The response of grandstands to dynamic crowd loads
}

\author{
B. R. Ellis, BSc, PhD, CEng, MIStructE, T.Ji, BSc, MSc, PhD, CEng, MIStructE, RA \\ and J. D. Littler, PhD
}

- This paper provides an overview of the response of grandstands to dynamic crowd loading. It summarizes the guidance which is currently available in the UK and how it has been developed. The interaction between structures and crowds is then considered for both jumping and stationary crowds. A model for jumping loads is given, and the frequency range for jumping and the dynamic crowd effect are discussed. Numerical modelling of grandstands and the determination of structural response are considered, together with possible structural modifications to improve dynamic behaviour. Next, the testing of structures is examined and the results from tests on a range of grandstands summarized, including the characteristics of empty structures and response to crowd loading during concerts and sports events. Finally, vibration serviceability levels are considered and some other design considerations discussed.

Keywords: building structures \& design; recreational facilities

\section{Introduction}

It is recognized that crowds of people can generate significant dynamic loads, especially when the crowd movement involves rhythmic jumping. This situation can occur with some types of dancing and aerobics. If the frequency of the loading is such that a resonant response of the structure occurs, excessive structural vibrations may result. This is an important factor for dance floors and other structures which may encounter similar loads at events like pop concerts.

2. Pop concerts can attract large audiences, and it is becoming increasingly common for sports stadia to be used for such events. Therefore, the grandstands at stadia may encounter a more severe loading regime than considered in their design. Also, many modern grandstands are responsive to dynamic loads, and hence the safety and serviceability of these structures may need to be checked. Although the main concern regarding dynamic loading relates to jumping, it has been questioned whether crowd behaviour at sports events could pose a similar problem. Indeed, there has been a recent directive for football stadia to be checked and those not complying with certain frequency requirements to be subjected to dynamic analysis. This may prove to be a significant problem for many football clubs, especially those with long-span cantilevered grandstands.

3. The authors have been involved with the subject area for many years, including the development of load models for jumping, the evaluation of structural response to such loads, testing empty grandstands and monitoring the response of grandstands during concerts and sports events. The purpose of this paper is to provide an overview of the topic of grandstands subject to dynamic crowd loading. This will illustrate how the subject has developed and what difficulties may still be encountered in trying to assess the safety and serviceability of existing and future grandstands.

4. In this paper, the fundamental frequency of grandstands is mentioned on a number of occasions. This is an important parameter, as it relates to the stiffness and mass of a structure, which are key items in design. Also, it is usually the fundamental mode which will be of concern when evaluating response to dynamic crowd loads. A structure will have many modes of vibration, often related to motion principally in one direction. Thus there may be modes which are primarily vertical motion, with the lowest-frequency mode being termed the fundamental vertical mode. The vertical modes are usually the most important ones for permanent grandstands, although it may not always be the fundamental mode of the whole grandstand which is critical. Similarly, there will be a range of modes for two horizontal directions, which are herein termed sway and front-to-back (FtoB), which are often the most important modes for temporary and retractable grandstands.

Information in UK codes and guides related to dynamic crowd loads

5. Guidance on dynamic crowd loads was included in the 1996 version of BS $6399^{1}$ specifically to take account of loads generated by repetitive dancing. The guidance considers the loads generated by the activity which are independent of the structure to which they are applied. The relevant section of the standard states:
Proc. Instn Civ. Engrs Structs \& Bldgs, 2000, 140 Nov., 355-365

Paper 12305

Written discussion closes 25 January 2001

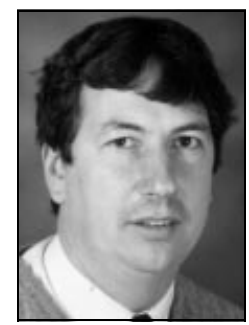

Brian Ellis,

Technical Director, Centre for Structural Engineering, Building Research Establishment Ltd, Watford

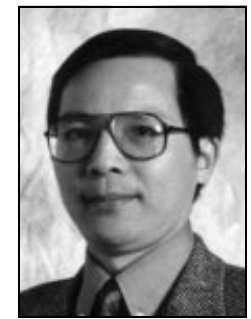

T. Ji, Lecturer, Department of Civil and Construction Engineering, UMIST, Manchester

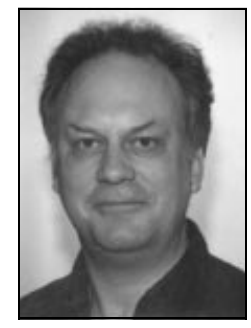

J. D. Littler, Head of Dynamic Performance, Centre for Structural Engineering, Building Research Establishment Ltd, Watford 
Dynamic loads will only be significant when any crowd movement (dancing, jumping, rhythmic stamping, aerobics etc.) is synchronized. In practice this only occurs in conjunction with a strong musical beat such as occurs at lively pop concerts or aerobics. The dynamic loading is thus related to the dance frequency or the beat frequency of the music and is periodical. Such crowd movement can generate both horizontal and vertical loads. If the synchronized movement excites a natural frequency of the affected part of the structure, resonance will occur which can greatly amplify its response.

Where significant dynamic loads are to be expected the structure should be designed either:

To withstand the anticipated dynamic loads; or

By avoiding significant resonance effects

To avoid resonance effects the vertical frequency should be greater than $8.4 \mathrm{~Hz}$ and the horizontal frequencies greater than $4.0 \mathrm{~Hz}$, the frequencies being evaluated for the appropriate mode of vibration of an empty structure.

To withstand the anticipated loads, reference is made to published work ${ }^{2}$ which is now summarized in a BRE Digest. ${ }^{3}$ It should be noted that the above frequency recommendations relate to safety assessments and not serviceability.

6. The event which focused interest on grandstand dynamics was the collapse of part of a temporary grandstand before a football match in Bastia, Corsica in 1992. The collapse killed 17 people and injured over 2500. An Institution of Structural Engineers working group was formed to examine temporary grandstands, and one of the topics covered was dynamic loading. This led to the DoE issuing guidance $^{4}$ in 1994 for checking temporary grandstands used at pop concerts, and here the frequency limits, which were later adopted by BS 6399, were introduced along with the alternative that safety may be achieved by ensuring that the structure will withstand the dynamic loads. Current guidance from the Institution of Structural Engineers on temporary grandstands ${ }^{5}$ refers to BS 6399.

7. A significant development occurred in 1997 when a revised version of the Guide to Safety at Sports grounds ${ }^{6}$ was issued; this guide is commonly known as the 'Green Guide'. Section (4.5) of the guide is related to dynamic response and states:

Dynamic load effects may be caused by

(a) Excitation by wind

(b) Excitation by the activities of spectators. Where a seating deck has a vertical frequency of less than $6 \mathrm{~Hz}$ or a sway frequency of less than $3 \mathrm{~Hz}$, a dynamic evaluation of the structure should be carried out giving due consideration to the mass of the spectators

(c) Excitation by the activities of spectators at grounds staging pop concerts or other events involving rhythmic activity, in which case the design loads may be greater than for category b).
Item (c) addresses similar concerns to BS 6399, but item (b) introduced a requirement for designated sports grounds which is apparently not related to rhythmic activity. No load model is provided for item (b) and no guidance is given on how to consider the mass of spectators. A subsequent publication ${ }^{7}$ concerned with grandstand dynamics provided further information, as did the discussion of that paper, ${ }^{8}$ which highlighted some remaining difficulties.

\section{Human actions on grandstands}

8. One area which has not always been fully understood is how people interact with structures. A common assumption has been that a crowd simply acts as a mass on a structure; however, the human body, besides having a mass, is flexible and can absorb energy. These are important characteristics of a dynamic system, and the simple mass model is not sufficient. In fact, there is a range of scenarios to consider, with the extreme cases being when the crowd is either all stationary or all jumping. The latter situation applies when evaluating response to jumping at pop concerts.

\section{A stationary crowd}

9. One experiment on a cantilevered grandstand provided a good illustration of the interaction between a grandstand and a stationary crowd. Here, the characteristics of a cantilevered grandstand were monitored before and during a rugby match. This involved recording the vertical response of a cantilever, from which a series of autospectra were derived using a Fourier transform procedure. Two autospectra are shown in Fig. 1, representing the vertical characteristics of the empty stand and the characteristics when full of seated people. If the crowd was acting solely as a mass, then the frequency of the stand would simply decrease with the increasing size of the crowd. The figure shows a different situation and suggests that the seated crowd acts as a sprung-mass system. It is also clear that the stationary crowd provides a significant increase in the damping capacity of the system. Further information on human-structure interaction, including laboratory tests and a simple mathematical model, is given in Ellis and $\mathrm{Ji}^{9}{ }^{9}$

10. Another informative example was encountered while monitoring a retractable grandstand. Here, measurements were made in all three orthogonal directions. In this experiment ${ }^{10}$ the crowd was asked to remain seated while one set of measurements was taken, and then asked to stand still while another set was taken. These showed significant differences in measured frequencies for the two situations (and also for the empty structure), demonstrating the inadequacy of representing the crowd solely as a mass. 


\section{A jumping crowd}

11. The critical load situation applies when all of a crowd is jumping, and here the crowd is not in contact with the structure for all of the time. In this situation, the crowd is acting solely as a load, with no effect on the structural characteristics, neither frequency nor damping. Thus, for this situation, it is the characteristics of the empty structure that are required to determine the structural response. This has important implications, because those who are jumping will not feel the vibrations and hence will not be aware of the vibrating structure even if large accelerations are generated. The jumpers will only be aware of the vibration levels when they stop jumping and start interacting with the structure. However, if the vibration levels were very high, they would inevitably affect the jumping.

\section{Dynamic crowd loads}

\section{Synchronous repetitive vertical loads}

12. The most severe loading conditions occur when the loading is both synchronized and periodic, and this is the situation considered in BS 6399. With synchronous repetitive loads, the basic model for vertical loads expressed as a Fourier series for one individual is

$$
F_{\mathrm{s}}(t)=G_{\mathrm{s}}\left(1 \cdot 0+\sum_{n=1}^{\infty} r_{n} \sin \left(\frac{2 n \pi}{T_{\mathrm{p}}} t+\varphi_{n}\right)\right)
$$

where $F_{\mathrm{s}}(t)$ is the dynamic load, $G_{\mathrm{s}}$ is the weight of the dancer, $r_{n}$ is the Fourier coefficient (or dynamic load factor) of the $n$th term, $n$ is the number of Fourier terms, $T_{\mathrm{p}}$ is the period of the jumping load, and $\varphi_{n}$ is the phase lag of the $n$th term. The Fourier terms in the summation are effectively the dynamic load, so when the movement stops the load reduces to the actual static load.

13. Although the expression of the load equation (1) has been used in other building codes and design guides, ${ }^{11-13}$ a difficulty has been the determination of Fourier coefficients $r_{n}$ and phase lags $\varphi_{n}$. A few Fourier coefficients have been determined by experiment, but no phase lags. Thus the load could not actually be expressed using equation (1), which has restricted theoretical investigations. These difficulties were removed when Ji and Ellis ${ }^{2}$ was published and the Fourier coefficients were determined.

14. The first six Fourier coefficients for three types of jumping are given in Table 1. These values enable equation (1) to be used. However, the frequency ranges which correspond to the various Fourier terms need to be identified, and these relate directly to the frequency range over which people can jump.

15. Figure 2 shows the force-time history

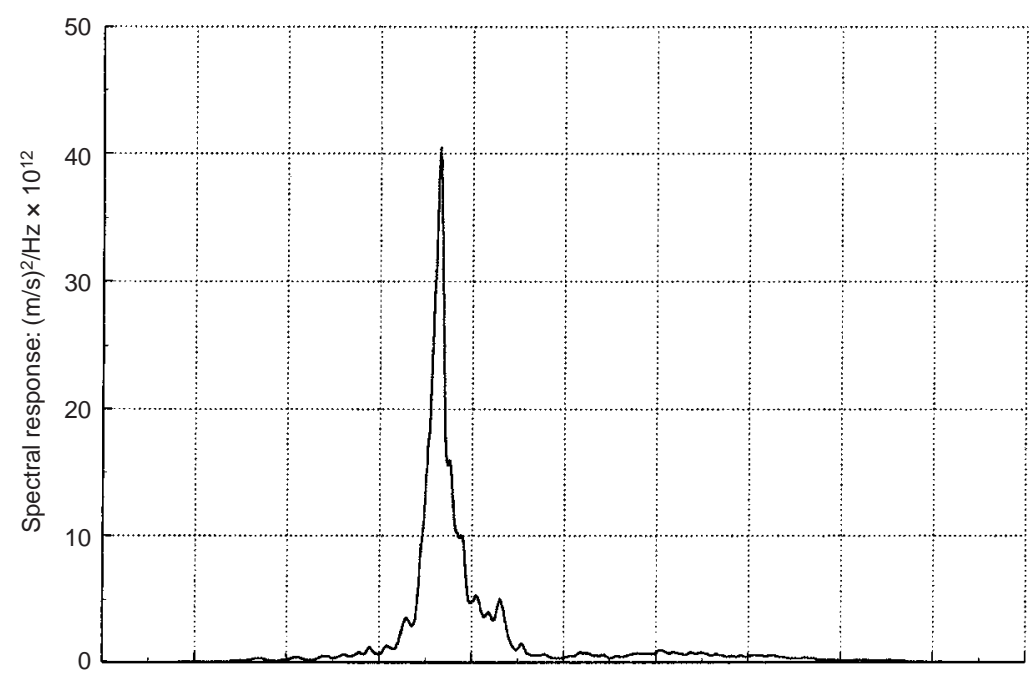

(a)

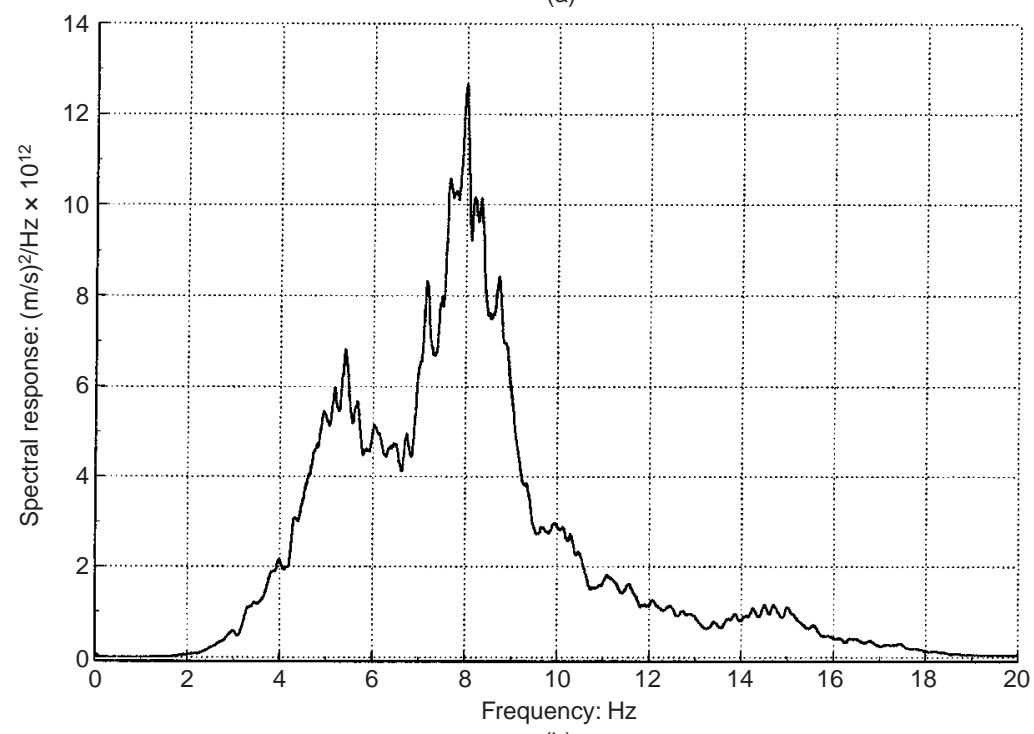

(b)

determined using equation (1), adopting 12 and 3 Fourier terms in the calculation. The figure illustrates two load cycles at $2 \mathrm{~Hz}$ of 'normal jumping' which has a contact ratio $(\alpha)$ of $1 / 3$, that is, for two-thirds of each cycle the body is not in contact with the structure. In the figure the force is normalized so that the body weight is unity $\left(G_{\mathrm{S}}=1\right)$. The peak load is $4 \cdot 61$ times the body weight, that is, $\pi / 2 \alpha$. It can be seen that the higher Fourier terms $(n>3)$ do not affect the peak load significantly. However, they may affect the structural response, depending on the ratio of the load frequency to structural frequency.

\section{Synchronous repetitive horizontal loads}

16. From measurements on a range of structures, the largest horizontal responses occur when jumping is encountered. Therefore it seems that a load model similar to equation (1) is appropriate, but omitting the term representing the static weight. It is evident that the horizontal load is much smaller than the
Fig. 1. Autospectra measured on a cantilever grandstand when empty (a) and full (b) 
ELLIS ET AL.

Table 1. Fourier coefficients and phase lags for different types of jumping

\begin{tabular}{l|c|c|c|c|c|c|c|c}
\hline Activity & Contact ratio: $\alpha$ & Coefficients & $n=1$ & $n=2$ & $n=3$ & $n=4$ & $n=5$ & $n=6$ \\
\hline $\begin{array}{l}\text { Low-impact } \\
\text { aerobics }\end{array}$ & $2 / 3$ & $r_{n}$ & $\frac{9}{7}$ & $\frac{9}{55}$ & $\frac{2}{15}$ & $\frac{9}{247}$ & $\frac{9}{391}$ & $\frac{2}{63}$ \\
& & $\varphi_{n}$ & $-\frac{\pi}{6}$ & $-\frac{5 \pi}{6}$ & $-\frac{\pi}{2}$ & $-\frac{\pi}{6}$ & $-\frac{5 \pi}{6}$ & $-\frac{\pi}{2}$ \\
\hline $\begin{array}{l}\text { Rhythmic } \\
\text { exercises, } \\
\text { high-impact } \\
\text { aerobics }\end{array}$ & $1 / 2$ & $r_{n}$ & $\frac{\pi}{2}$ & $\frac{2}{3}$ & 0 & $\frac{2}{15}$ & 0 & $\frac{2}{35}$ \\
\hline $\begin{array}{l}\text { Normal } \\
\text { jumping }\end{array}$ & $1 / 3$ & $\varphi_{n}$ & 0 & $-\frac{\pi}{2}$ & 0 & $-\frac{\pi}{2}$ & 0 & $-\frac{\pi}{2}$ \\
\hline
\end{tabular}

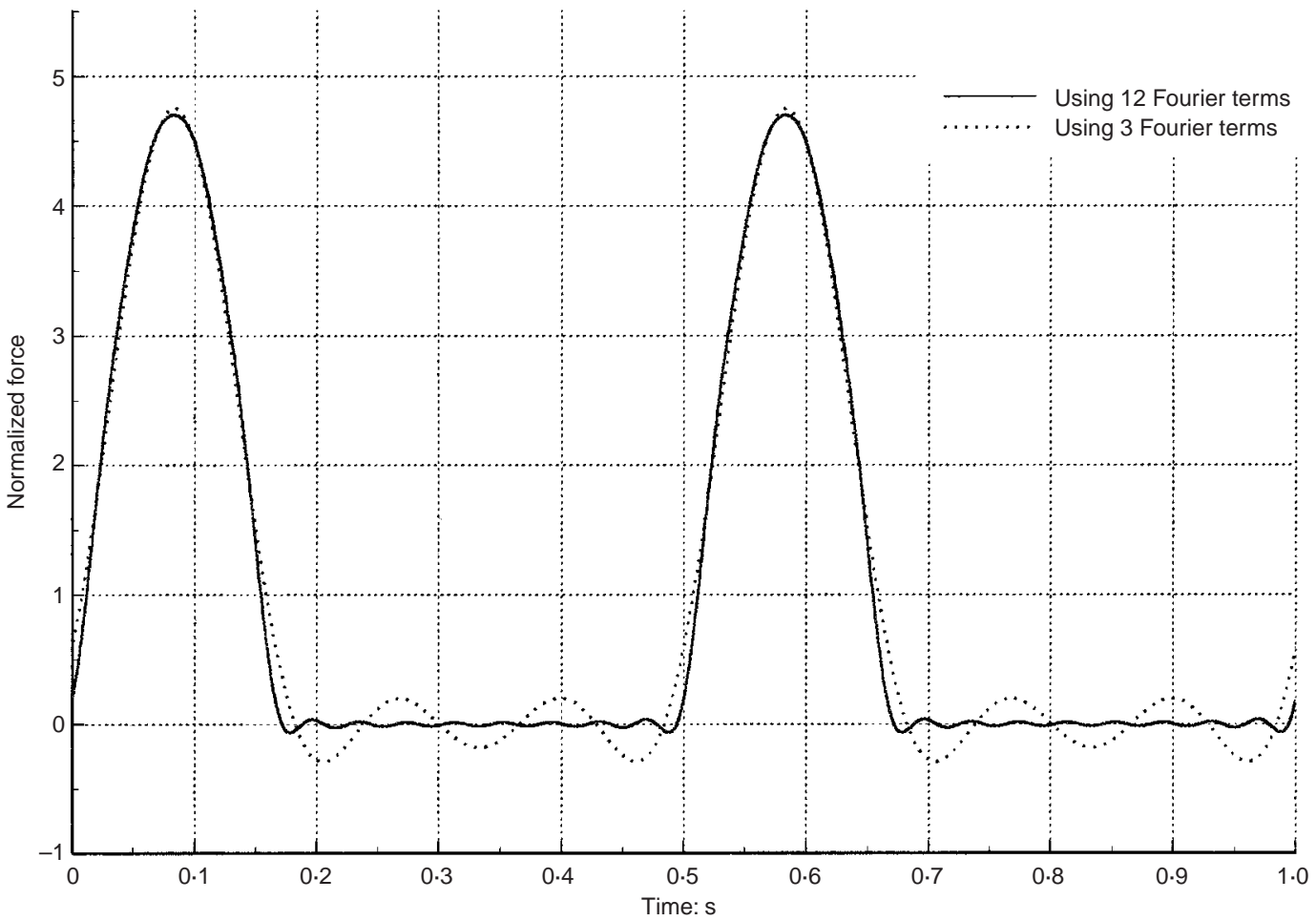

Fig. 2. Load-time history for normal jumping at $2 \mathrm{~Hz}$

vertical load, and from a few measurements the horizontal load appears to be about $7-10 \%$ of the vertical load; however, more data are needed to verify these values.

\section{Frequency range for jumping}

17. In BRE Digest 426 it was suggested that the frequency range for individuals jumping is approximately $1.5-3.5 \mathrm{~Hz}$, but for a crowd the higher-frequency jumping cannot be sustained and an upper limit of $2.8 \mathrm{~Hz}$ is more realistic. However, it was recognized that further detailed information was required, examining the actual range at which people can jump comfortably and where a crowd can achieve some degree of coordination.
18. To investigate the range, the beat frequencies of a sample of 210 modern popular songs were examined. ${ }^{14}$ The investigation included tests to assess the frequency ranges for coordinated dance-type loads for individuals, small groups (aerobics) and large groups (pop concerts). Some of the conclusions from this work were that the frequency ranges are:

(a) $1 \cdot 2-2 \cdot 8 \mathrm{~Hz}$ for an individual jumping

(b) $1 \cdot 5-2 \cdot 5 \mathrm{~Hz}$ for a small group jumping (aerobics) with some degree of coordination

(c) $1 \cdot 8-2 \cdot 3 \mathrm{~Hz}$ for a large group jumping (pop concerts) with some degree of coordination. 


\section{The dynamic crowd effect}

19. When a dynamic assessment of a grandstand is to be conducted, the actual load density and distribution of the crowd should be considered, rather than the static design load. Consideration should also be given to the dynamic crowd effect, which describes the attenuation of load due to the imperfect coordination between individuals in a group. Therefore, the load model for a group of people becomes

$$
\begin{aligned}
& F(x, y, t) \\
& \quad=G(x, y)\left[1 \cdot 0+C_{\mathrm{e}} \sum_{n=1}^{\infty} r_{n} \sin \left(\frac{2 n \pi}{T_{\mathrm{p}}} t+\varphi_{n}\right)\right]
\end{aligned}
$$

where $F(x, y, t)$ is the dynamic crowd load, $G(x, y)$ is the load density and distribution of the crowd, and, $C_{\mathrm{e}}$ is the dynamic crowd factor.

20. The dynamic crowd effect depends on the coordination of the people in the group, the type of dancing and the beat frequency of the music. A theoretical investigation where the phase lag between individuals was treated as a random variable obeying a normal distribution, ${ }^{15}$ showed a one-third reduction of the crowd loads for a large group of people. As only the first Fourier term was considered in this study, the reduction is likely to be conservative. The principles used in this investigation are applicable to studying the effects for higher Fourier terms.

21. The Danish code ${ }^{11}$ considers different factors for different crowd sizes and different Fourier terms. However, it is difficult to obtain good experimental data to validate such methods, in which both phase lags and contact ratios may vary, and where the attitude and experience of the group are important. Work is currently being undertaken to provide a consistent European approach.

\section{Theoretical studies}

\section{Numerical modelling}

22. The finite element method is useful for analysing the dynamic behaviour of a grandstand, in particular for calculating the frequencies and mode shapes using eigenvalue analysis. Also, the method can be used for calculating the response of the structure subject to rhythmic crowd loads, although it will require a significant amount of computation and an estimate of the damping value.

23. Either a permanent or a temporary grandstand can be considered as a plane frame repeated several times in the longitudinal direction linked by horizontal elements. Therefore, it is straightforward to model a grandstand as a plane frame initially. However, not many grandstands have been studied beyond the basic frame models. Also, the continuity of the horizontal elements is an important factor which influences structural behaviour.

24. Two investigations using numerical modelling have been conducted at UMIST to examine the structural dynamic behaviour of permanent grandstands. One examined the effects of different finite element models for evaluating the dynamic behaviour of a grandstand, and included the effect of mesh sizes, seating decks and bracing arrangements. ${ }^{16}$ The other investigated the dynamic behaviour of a grandstand as it was extended from a plane frame to an open-ended stand (with and without end supports) and then to an enclosed stand. ${ }^{17}$ Fig. 3 shows a model of an enclosed grandstand. $^{18}$

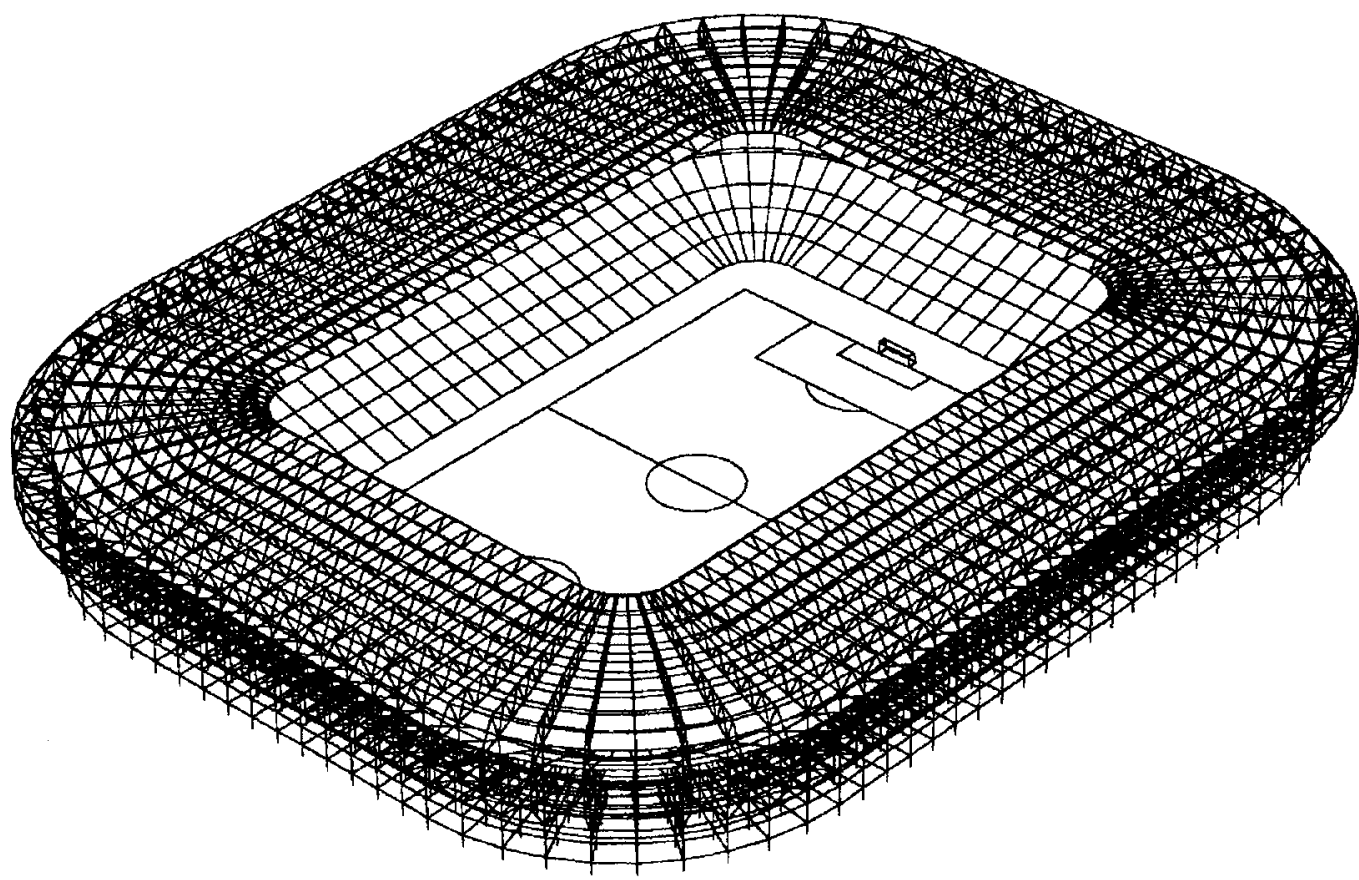

Fig. 3. A model of an enclosed grandstand 
25. Some conclusions drawn from these studies can be summarized as follows.

(a) Relatively coarse meshes can be used for eigenvalue analysis.

(b) The eccentricities between seating decks and supporting elements should be considered; these effectively increase the stiffness or frequency of cantilever tiers and reflect the actual structure.

(c) The vertical and $\mathrm{FtoB}$ vibrations are often coupled, which may explain why vertical crowd loads cause the FtoB vibration as observed in practice.

(d) The roofs of grandstands may have lower frequencies than those of the tiers.

(e) The fundamental mode of the enclosed model shows a torsional movement, while that of the corresponding open-ended model gives a sway movement.

$(f)$ Bracing systems play a significant role in increasing structural stiffness in sway and torsion.

(g) Bracing should also be arranged in the roof to increase its sway stiffness.

\section{Determination of structural response}

26. An analytical solution was developed to calculate floor vibrations induced by dynamic crowd loads, ${ }^{2}$ and this is applicable to grandstands subject to the same type of loading.

27. The assumptions adopted in the solution were as follows.

(a) The dynamic crowd load can be expressed as a product of two functions in the space and time domains respectively, that is

$$
F(x, y, t)=G(x, y) S(t)
$$

(b) The structural response is dominated by one mode (usually its fundamental).

28. Equations 1 and 2 satisfy the first assumption. The second assumption limits the application of the method to structures whose responses are primarily in one mode. Modifications of the method can be made when the contributions from other modes need to be considered. However, from experiments and numerical modelling, it appears that the behaviour of a wide range of grandstands is dominated by the response in a single mode.

29. If the structure is subjected to uniformly distributed human loads, i.e. $G(x, y)$ becomes a constant, and the maximum displacement and acceleration can be expressed as follows:

$$
\begin{aligned}
A & =\frac{B G}{m \omega^{2}}\left[1+C_{\mathrm{e}} D^{\mathrm{d}}\left(\omega, \omega_{\mathrm{p}}, \zeta, t, \alpha\right)\right] \\
\ddot{A} & =\frac{B G}{m} C_{\mathrm{e}} D^{\mathrm{a}}\left(\omega, \omega_{\mathrm{p}}, \zeta, t, \alpha\right)
\end{aligned}
$$

where $m$ is the structural mass density, $D^{\mathrm{d}}$ and $D^{\text {a }}$ are dynamic magnification factors for displacement and acceleration, $\omega$ is the circular frequency of the mode being considered (i.e. $2 \pi$ times the natural frequency), $\omega_{\mathrm{p}}$ is the circular frequency of the load, $\zeta$ is the damping ratio, and $B$ is a structural factor which relates to the fundamental mode and depends on the type of structure and boundary conditions. Factors for several simple structures have been provided. ${ }^{2}$

30. The structural response to dynamic crowd loads can be evaluated using equation (4), but it requires a computer implementation for evaluating the dynamic magnification factors. A worked example using MATHCAD is provided in BRE Digest 426. A simple graphical method for performing these calculations has been developed and is currently being prepared for publication.

\section{Structural improvements}

31. When an existing grandstand fails to meet the required safety or serviceability levels, the question of whether the structure can be modified arises. If the problem just relates to pop concerts, then a solution may simply be to select an alternative venue. However, some structures can be modified to increase their stiffness, and one such situation is considered here.

32. With temporary grandstands it is usually the taller structures which have the lower horizontal frequencies, and here the arrangement of the bracing elements is critical. Temporary grandstands usually have a number of bays and storeys. The selection of the layout of a temporary grandstand is constrained by the site, visibility and required size, while the arrangement of bracing members of the grandstand has few restrictions. Different bracing configurations and patterns will provide different lateral stiffnesses or frequencies, even if the same number of bracing members is used. Criteria for arranging bracing members to achieve a large lateral stiffness have been considered, ${ }^{19}$ and the recommendations were as follows.

(a) Members in different storeys should be provided from the top to the support (base) of the structure.

(b) Members in different storeys should be directly linked where possible.

(c) Members should be linked in a straight line where possible.

(d) Members at the top of adjacent bays should be directly linked where possible.

(e) If extra bracing members are required, they should adopt the above criteria.

33. As an example, Fig. 4 shows an idealization of a typical bracing system and a rearrangement of the bracing members following the above recommendations. The rearrangement uses less bracing members than the original scheme $(81 \%)$ but achieves an increase 
in frequency of $69 \%$ and an increase in stiffness of $184 \%$.

34. While the recommendations apply to a wide range of structures, there are some structures where simple alterations are not available. For example, retractable stands are usually flexible in the FtoB direction, and while extra bracing could be added, it would affect their functionality. Also, large cantilever grandstands provide good viewing of sports events, but their design is far from ideal for resisting vertical dynamic loads. In general, the larger the span of the cantilever, the lower the fundamental frequency, and the more severe the potential dynamic response at events like pop concerts. Here, structural modifications are difficult if the basic cantilever system is to be maintained.

\section{Dynamic testing}

35. Performing dynamic tests on existing grandstands can provide useful information. BRE has developed test methods and facilities and used them on a wide range of grandstands. The experiments include measurement of frequencies, damping, modal stiffness and mode shapes, and monitoring response due to dynamic crowd loads at a variety of events.

\section{Measurement of modal characteristics}

36. A person jumping once on a structure will excite the fundamental vertical mode, and recording and analysing the response from a vertically mounted transducer will enable the frequency to be determined. The measurement of vertical frequencies at a range of positions will also help us to understand how the structure behaves; for example, a cantilevered grandstand in which the main beams are not positively connected to adjacent beams will be quite different from one where the beams are rigidly connected and monolithic behaviour of the structure occurs.

37. The fundamental horizontal frequencies can be more difficult to measure, especially for very large structures. One method of taking these measurements at outdoor venues is to record the structure's response to ambient (wind) excitation and derive the frequency from the recorded response. For very large structures on a relatively calm day, this requires the use of a sensitive measurement system, and here BRE uses laser measurements.

38. To define any one mode fully, it is necessary to determine its frequency, damping, stiffness and mode shape. This is undertaken using a forced vibration test. Fig. 5 shows one of the BRE exciters on a cantilever stand, and Fig. 6 shows a measured response spectrum; the continuous curve which is fitted to the measured points is defined by stiffness, frequency and damping.

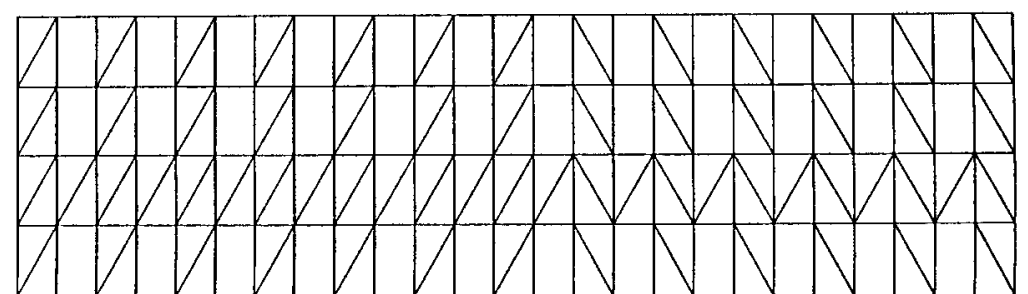

(a)

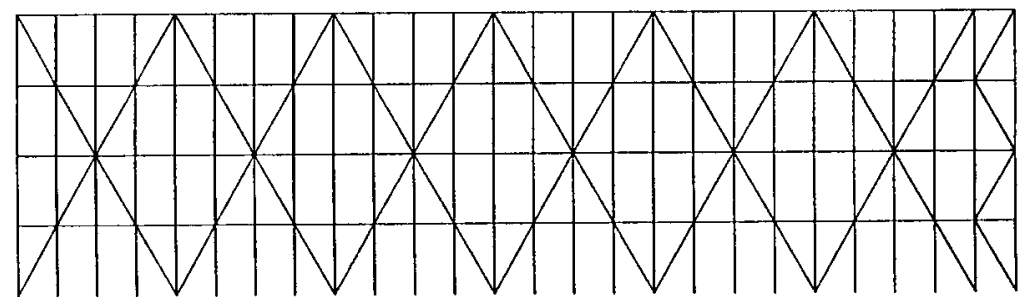

(b)

Fig. 4. Bracing arrangements for a temporary grandstand: (a) idealization of a typical bracing system; (b) arrangement of bracing following the proposed criteria

Monitoring response to crowd loading

39. To monitor reaction to crowd loading, the structural response is recorded and analysed. For serviceability it is not sufficient just to consider the peak acceleration, as both the frequency content and duration are important.

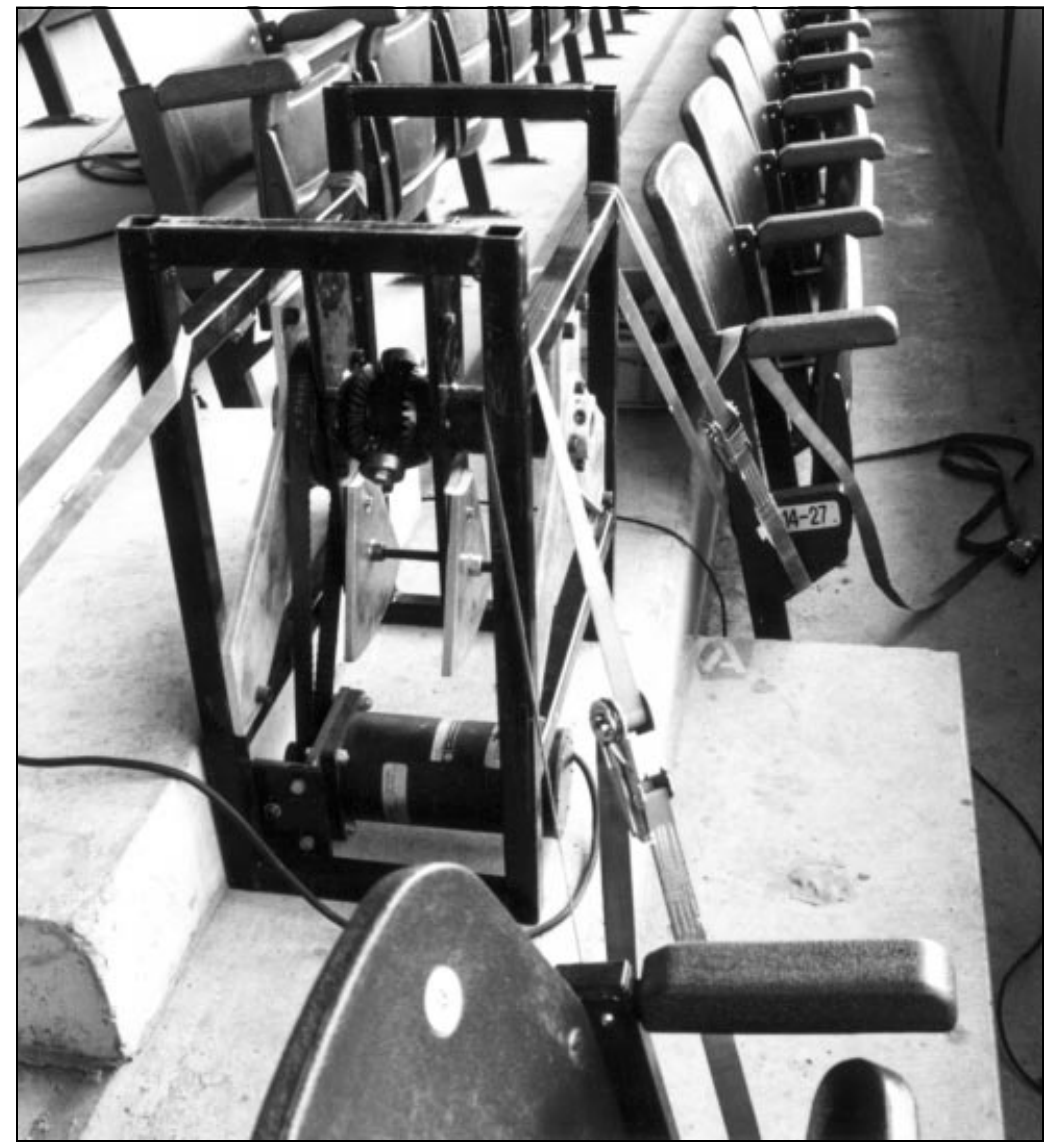

Fig. 5. One of BRE's vibration generators on a cantilever grandstand 


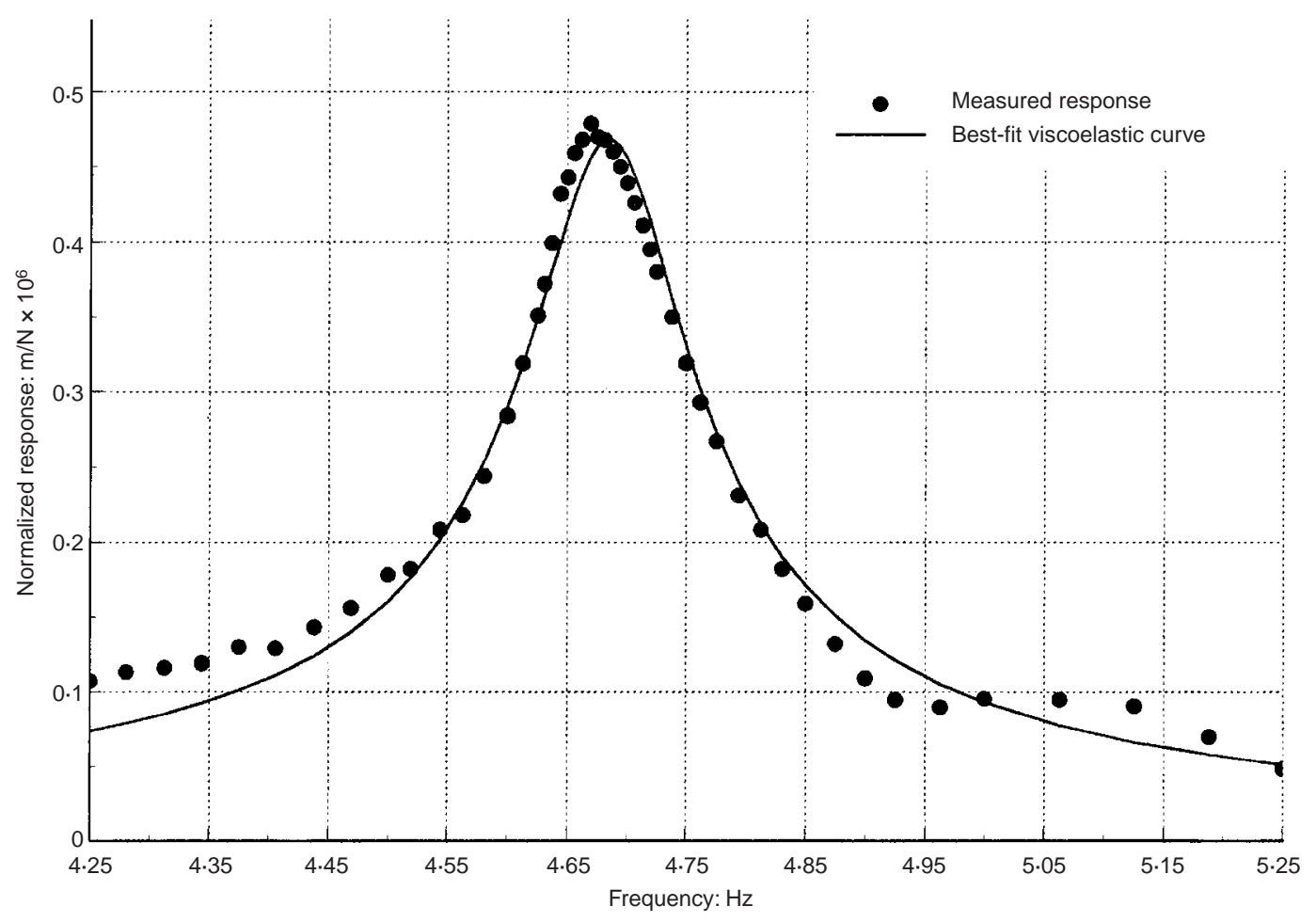

Fig. 6. Vertical response spectrum for a cantilever grandstand
Early tests evaluated peak accelerations and those below $10 \mathrm{~Hz}$, but current work adopts the BS $6841^{20}$ frequency weighting recommendations and considers vibration dose values. The data are also analysed to examine the resonance frequency of the structure and how it changes with the size of crowd, as well as to examine the response to any specific event. Fig. 1 shows spectra for an empty and full grandstand, but neither of these situations involves a large dynamic load. Fig. 7 shows autospectra for vertical and horizontal motion measured on a cantilevered grandstand with a crowd jumping. Here the jumping frequency and its harmonics can clearly be identified, and this form of response is consistent with that expected, given the load model (equation (2)).

Results from tests on cantilevered grandstands 40. BRE has conducted detailed tests on eleven cantilevered stands. ${ }^{21}$ The capacities of the cantilevered tiers range from 600 to over 10000 seats. Except for a rugby stadium, all the stands had vertical frequencies below $8 \cdot 4 \mathrm{~Hz}$, with a range from $2 \cdot 08$ to $6.79 \mathrm{~Hz}$ and damping from $1 \cdot 3 \%$ to $4 \cdot 7 \%$. Stands at 17 of the 18 major English football stadia examined had vertical modes under $6 \mathrm{~Hz}$, and of these three were under $3 \mathrm{~Hz}$ (some of these were subjected to limited tests).

41. Ten stands were monitored during public events. Five were monitored during sporting events and five during pop concerts.

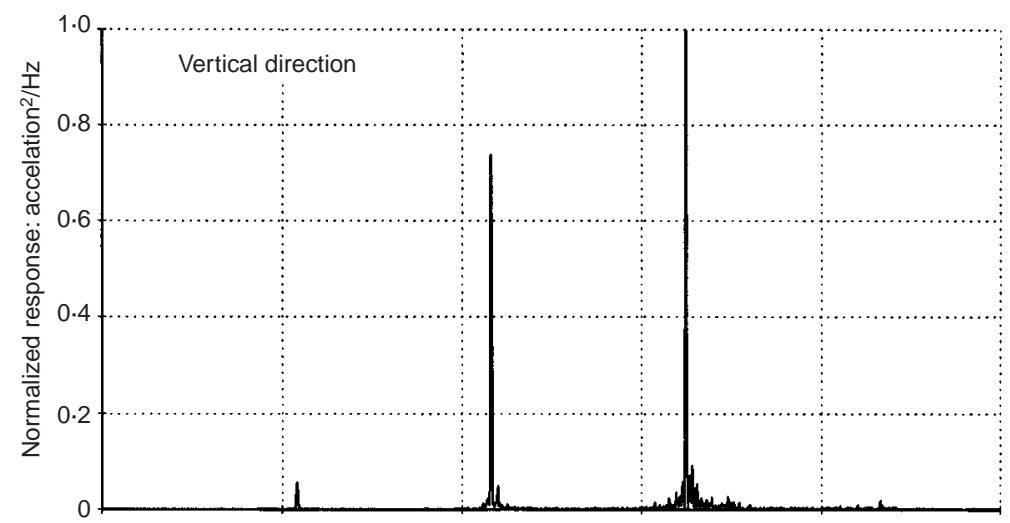

(a)

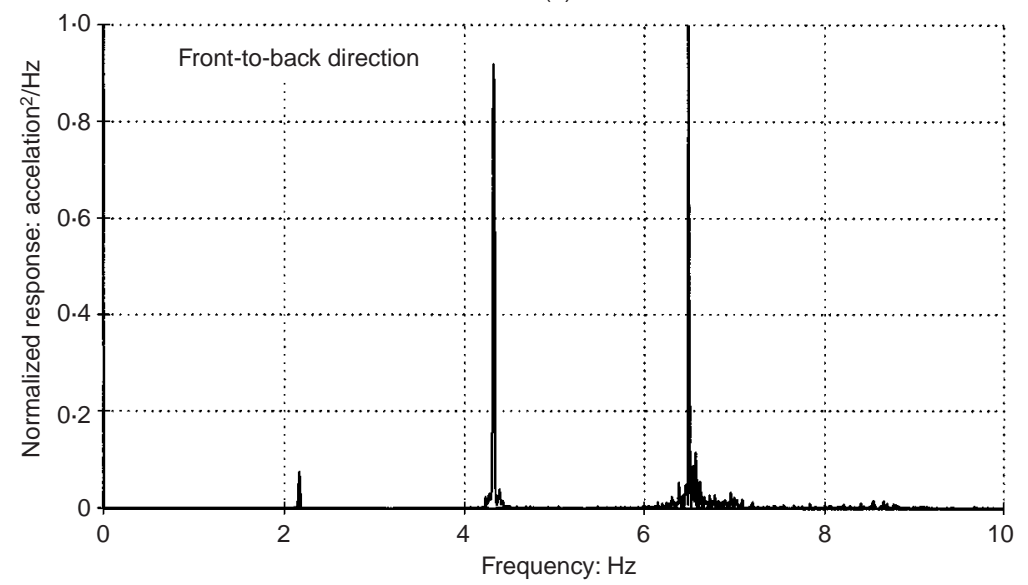

(b)

Fig. 7. Autospectra measured on a cantilever grandstand during a pop concert: (a) vertical direction; (b) front-to-back direction 
The maximum peak acceleration during sporting events ranged from $2.5 \%$ to $52 \% \mathrm{~g}$. The maxima occurred when the crowd reacted to an event on the pitch, such as a goal being scored. The frequencies at which the peak accelerations occurred were relatively high. For example, when the record containing the peak acceleration of $52 \% \mathrm{~g}$ was passed through a $10 \mathrm{~Hz}$ lowpass filter, the peak response reduced to $2 \cdot 4 \% \mathrm{~g}$. These vibrations appeared to be of little concern to the crowd.

42. The responses measured during concerts were quite different. Motion of the stands was perceptible at all of the concerts, and in some cases this motion was clearly perceptible and would be 'disturbing' under the serviceability classification system given in 'Serviceability limits' below. While several people remarked on the movement of two of the stands, nobody questioned the integrity of the stands. The maximum single peak accelerations ranged from $3 \cdot 1 \%$ to $16 \cdot 5 \% \mathrm{~g}$. Almost all of the response occurred at the beat frequency of the music or one of its harmonics. When the data were passed through a $10 \mathrm{~Hz}$ low-pass filter, peak responses of up to $8.2 \% \mathrm{~g}$ were obtained.

\section{Results from tests on temporary demountable grandstands}

43. The 50 demountable stands tested by BRE were of 15 different types, with capacities from 243 to 3500 seats. $^{22}$ Only one stand had a vertical mode below $8 \cdot 4 \mathrm{~Hz}($ at $7 \cdot 9 \mathrm{~Hz})$. Table 2 shows the distribution of horizontal natural frequencies (to the nearest $0 \cdot 1 \mathrm{~Hz}$ ). The measured damping values for the fundamental sway modes were in the range $1 \cdot 5-4 \cdot 5 \%$ critical; those for the fundamental FtoB modes were in the range $2 \cdot 2-6 \cdot 0 \%$ critical, with one exception, where a value of about $17 \%$ critical was obtained.

44. Twenty-three stands were monitored when fully occupied during different events. Six of these events were pop or rock concerts and one was a drama festival. The remaining 16 were sporting events. Table 3 gives the maximum measured peak accelerations. For the tests performed after 1992, the maximum accelerations with frequency components below $10 \mathrm{~Hz}$ are also given.

45. Motion of some of the stands monitored during pop and rock concerts was both perceptible and visible in certain songs when sections of the crowd were dancing.

\section{Results from tests on retractable grandstands}

46. Six retractable stands have been tested by BRE. ${ }^{10}$ Two of the stands hold approximately 1400 people, two hold around 500 people and the remaining two have capacities of about 200 and 100 . Only one of the retractable stands had a vertical mode below $8 \cdot 4 \mathrm{~Hz}($ at $7 \cdot 6 \mathrm{~Hz}$ ). Table 4 shows the distribution of horizontal
Table 2. Fundamental horizontal frequencies of temporary demountable grandstands

\begin{tabular}{l|c|c}
\hline \multirow{2}{*}{ Frequency: $\mathrm{Hz}$} & \multicolumn{2}{|c}{ Number of stands } \\
\cline { 2 - 3 } & Sway & FtoB \\
\hline Under $3 \cdot 0$ & 15 & 10 \\
$3 \cdot 0-3 \cdot 9$ & 17 & 13 \\
$4 \cdot 0-4 \cdot 9$ & 13 & 9 \\
$5 \cdot 0$ or over & 5 & 18 \\
\hline
\end{tabular}

Table 3. Maximum measured peak accelerations (\%g)

\begin{tabular}{l|c|c|c|c}
\hline \multirow{2}{*}{ Event } & \multicolumn{2}{|c|}{ Unfiltered } & \multicolumn{2}{c}{ Filtered at $10 \mathrm{~Hz}$ (low-pass) } \\
\cline { 2 - 5 } & Vertical & Horizontal & Vertical & Horizontal \\
\hline Concerts & $46-243$ & $41-606$ & $9 \cdot 2-21 \cdot 4$ & $6 \cdot 9-129$ \\
Soccer & $39-460$ & $29-282$ & $7 \cdot 1-41 \cdot 8^{*}$ & $2 \cdot 0-13 \cdot 2$ \\
Motor racing & $39-96$ & $34-160$ & $1 \cdot 0-22 \cdot 4$ & $1 \cdot 0-6 \cdot 1$ \\
Golf & $11 \cdot 2-109$ & $6 \cdot 1-42 \cdot 8$ & $2 \cdot 0-7 \cdot 1$ & $0 \cdot 5-1 \cdot 0$ \\
Boxing & 84 & 29 & - & - \\
Horse racing & 32 & 10 & $4 \cdot 1$ & $1 \cdot 0$ \\
Tennis & $44 \cdot 8$ & $16 \cdot 3$ & $3 \cdot 1$ & $1 \cdot 0$ \\
Drama festival & $19 \cdot 4$ & $50 \cdot 0$ & $1 \cdot 0$ & $1 \cdot 0$ \\
\hline
\end{tabular}

* $15 \mathrm{~Hz}$ filter used.

Table 4. Fundamental horizontal natural frequencies of retractable stands

\begin{tabular}{l|c|c}
\hline \multirow{2}{*}{ Frequency: $\mathrm{Hz}$} & \multicolumn{2}{|c}{ Number of stands } \\
\cline { 2 - 3 } & Sway & FtoB \\
\hline $2 \cdot 0-2 \cdot 9$ & 1 & 4 \\
$3 \cdot 0-3 \cdot 9$ & 2 & 2 \\
$4 \cdot 0-4 \cdot 9$ & 3 & - \\
\hline
\end{tabular}

natural frequencies for the stands (to the nearest $0 \cdot 1 \mathrm{~Hz}$ ).

47. The measured damping values on the different stands varied widely. Values between $0.6 \%$ and $9 \%$ were measured in the sway direction, and between $3 \cdot 2 \%$ and $11 \%$ in the FtoB direction.

48. Three of the retractable stands were monitored during pop concerts. Motion was perceptible on all but one of the retractable stands (where there was little audience response). The movement of the large stand could be seen quite easily. Several people asked to be relocated off the stand because they felt unsafe, and some stewards were concerned by how much they could see the stand moving.

\section{Design considerations}

\section{Serviceability limits}

49. In order to determine whether a structure will be safe under a given load, the maximum deflection and stresses need to be evaluated and compared with design values. 
For serviceability assessments related to human perception of vibrations, it is the acceleration levels which must be determined. However, the question of what are acceptable vibration levels must then be considered. A significant amount of work has been conducted on what vibration levels can be perceived, and it is known that the frequency of the vibration is important, so the UK standard ${ }^{20}$ related to perception of vibrations provides frequencyweighting recommendations for motion in various directions. In addition, the number of cycles of the vibration is important when assessing tolerance to such vibrations. Hence, a vibration level which is encountered over one cycle (i.e. impulsive) will have a different effect from a vibration level which is continuous.

50. For guidance on acceptable vibration levels in grandstands subject to jumping loads, it seems appropriate to examine data obtained at such events. A number of experiments have been undertaken in Germany, ${ }^{23}$ and recommendations for low-frequency vibration are given in Table 5.

51. From Table 5 it can be seen that $35 \% g$ is a significant limit, as above that level panic may occur. However, below this level the vibration levels will certainly be felt by anyone remaining stationary on the structure. The authors experienced vibrations of $0 \cdot 15 g$ on one structure and this certainly felt uncomfortable, even with knowledge of the source of the vibrations. Limiting vibration levels to $0.05 \mathrm{~g}$, which has been suggested elsewhere, seems inappropriate, as this level is exceeded at most events.

52. It is also likely that visible motion of a cantilever and items fixed to it would be of concern to those immediately below the structure.

\section{For pop concerts}

53. A coordinated crowd jumping can generate large loads, and if the jumping frequency is such that a resonant structural response occurs, then the motion can be significant. Experience has shown that most engineers who first encounter this form of loading have significant reservations about its severity; however, videos of grandstands moving and large crowds jumping in time at pop concerts provide compelling support. Nevertheless, the critical situation where the members of a crowd are all jumping at the correct frequency to generate a resonant structural response is rare. This situation may occur at a lively pop concert in which a motivated audience is actively encouraged to jump for a number of songs with a range of beat frequencies (the timing usually being aided by large video screens); however, it still requires a responsive structure, usually one with a low fundamental frequency and low damping. If this combination is encountered,
Table 5. Reaction to various peak acceleration levels on grandstands

\begin{tabular}{l|l}
\hline $\begin{array}{l}\text { Vibration } \\
\text { level }\end{array}$ & Reaction \\
\hline$<5 \% g$ & Reasonable limit for passive persons \\
$<18 \% g$ & Disturbing \\
$<35 \% g$ & Unacceptable \\
$>35 \% g$ & Probably causing panic \\
\hline
\end{tabular}

then both safety and serviceability need to be checked. If safety is a potential problem, then it would not be wise simply to monitor the structure, with a view to stopping the music if a pre-set vibration level is exceeded, as a resonant vibration can build up very quickly and those jumping do not feel the structural vibrations which might otherwise limit the activity. As this is a rare event, it should not be considered in combination with other forms of live loading.

\section{For sports events}

54. Measurements have shown that some stadia have relatively lively stands, so there are potential problems at pop concerts. But could other situations be encountered, say at sports events, where similar loads are generated? Generally, for events like football, the crowd motion is not coordinated, even when a goal is scored and the crowd jumps to its feet. But this is not repetitive motion, so there can be no resonant build-up of structural response. Crowds at sporting events stamping in time to chanting can produce a succession of impacts at roughly equal intervals; however, without music, crowds do not seem to be very good at keeping to a particular rhythm. It is also normal for pop music to be played before and after games. But, given the low probability of the members of a football crowd all jumping to the music, at the correct frequency to generate resonance on a responsive stand, it may seem appropriate to avoid the situation through active management of the music being broadcast rather than choosing the expensive option of structural alterations. However, it is important to alert stadium managers to potential problems of this nature.

\section{Conclusions}

(a) Current UK guidance suggests that two forms of dynamic load should be considered for grandstands, one for activities of spectators at normal sports events, and a second for rhythmic crowd loads (jumping) at events like pop concerts.

(b) A stationary crowd will interact with the structure, whereas a jumping crowd will act solely as a load. For analysis of response to jumping, it is the characteristics of the empty structure that are important. 
(c) A load model is available which defines different activities involving jumping. However, further work is required on the dynamic crowd effect which describes the lack of coordination between individuals in a group.

(d) Finite element analysis is one method for calculating the dynamic characteristics of a structure, although the damping value will have to be estimated. This enables structural dynamic response to be evaluated, either using the finite element representation or a simple one-degree-of-freedom analysis. If excessive vibrations are encountered then some structures, like temporary grandstands, can be improved by using a more efficient bracing system, whereas others, like cantilever grandstands, may be more difficult to modify.

(e) Dynamic testing can be undertaken on existing stands, examining both the structural characteristics and structural response to crowd loading. The results from a range of tests on various types of grandstands are summarized to provide an indication of the behaviour of existing structures. For new structures where the dynamic characteristics have been calculated, it would be wise to take measurements following construction to verify the assumptions adopted in the analysis.

( $f$ ) Although the current UK guidance is concerned with safety, it is clear that serviceability related to human tolerance of the vibration levels is important. Work has been undertaken in Germany specifically on vibrations of grandstands, and the recommendations from that work, which are summarized herein, appear to be the best guidance currently available.

\section{Acknowledgments}

The authors would like to acknowledge the UK Department of the Environment, Transport and the Regions for supporting much of BRE's work, the EPSRC for supporting the UMIST research project on 'Design for safety of structures subject to dance-type loads', and the many owners and managers of grandstands for permission to undertake the experimental work.

\section{References}

1. British Standards Institution. Loading for Buildings. BSI, 1996, BS 6399: Part 1.

2. Ji T. and Eluis B. R. Floor vibration induced by dance type loads: theory. The Structural Engineer, 1994, 72, No. 3, 37-44.

3. Building Research Establishment. The Response of Structures to Dynamic Crowd Loads.
Construction Research Communications Ltd, London, October, 1997, BRE Digest 426.

4. Department of the Environment. Interim guidance on temporary grandstands. The Structural Engineer, 1994, 72, No. 15, August, 249-250.

5. Institution of Structural Engineers. Temporary Demountable Structures-Guidance on Design, Procurement and Use. SETO, London, 1999, pp. 77-86.

6. Scottish Office and Department of National Heritage. Guide to Safety at Sports Grounds, 4th edn. The Stationery Office, London, 1997.

7. Reid W., Dickie J. and Wright J. Stadium structures: are they excited? The Structural Engineer, 1997, 75, No. 22, 383-388.

8. Discussion on Stadium structures: are they excited? The Structural Engineer, 1998, 76, No. 13, 258-261.

9. Ellis B. R. and Ji T. Human-structure interaction in vertical vibrations. Structures and Buildings, 1997, 122, 1-9.

10. LitTler J. D. Retractable Grandstands: Dynamic Response. Building Research Establishment, Watford, 2000, BRE IP 4/00.

11. Danish Code, DS410: 1998, Code of practice for loads for the design of structures.

12. Bachman H. Vibration Problems in Structures: Practical Guidelines, Birkhäuser Verlag Basel.

13. International Standards Organization. Bases for Design of Structures - Serviceability of Build ings against Vibration. Geneva, ISO, 1992, ISO 10137.

14. Ginty D., Derwent J. M. and Ji T. The frequency range and distribution of dance type loads. Accepted for publication in The Structural Engineer.

15. Ji T. and Eluis B. R. Evaluation of dynamic crowd effects for dance type loads. Colloquium on Structural Serviceability of Buildings, Goteborg, 1993, 165-172.

16. EL-Houry M. The Effect of FE Modelling on the Structural Dynamic Behaviour of Grandstands. MSc dissertation, UMIST, 1999.

17. Mandal P. and Ji T. The dynamic behaviour of a grandstand - from a 2D frame to a complete 3D structure. To be submitted to The Structural Engineer.

18. Chong K. T. Design of a Grandstand. UMIST, Manchester, 1998, Project Report.

19. Ji T. and ElLis B. R. Effective bracing systems for temporary grandstands. The Structural Engineer, 1997, 75, No. 6, 95-100.

20. British Standards Institution. Guide to Measurements and Evaluation of Human Exposure to Whole-body Mechanical Vibration and Repeated Shock. BSI, 1987, BS 6841.

21. Littler J. D. Permanent Cantilever Grandstands: Dynamic Response. Building Research Establish ment, Watford, 2000, BRE IP 5/00.

22. LitTleR J. D. Temporary Demountable Grandstands: Dynamic Response. Building Research Establishment, Watford, 2000, BRE IP 3/00.

23. KASPERSKi M. Actual problems with stand structures due to spectator-induced vibrations. EURODYN'96, Florence, 1996, 455-461.

Please email, fax or post your discussion contributions to the secretary: email: wilson_1@ice.org.uk; fax: + 44 (0)20 7799 1325; or post to Lesley Wilson, Journals Department, Institution of Civil Engineers, 1-7 Great George Street, London SW1P 3AA. 